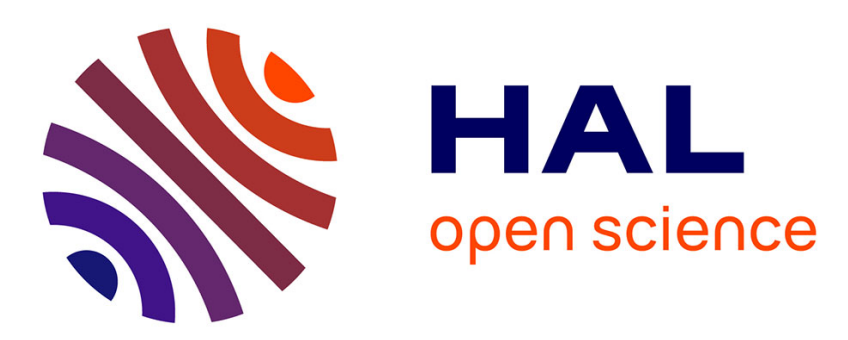

\title{
Black Phosphorus for Directed Molecular Assembly with Weak Electronic Coupling
}

Mehdi Bouatou, Rishav Harsh, Cyril Chacon, Yann Girard, Vincent Repain, Amandine Bellec, Sylvie Rousset, Alexander Smogunov, Yannick J Dappe, Jérôme Lagoute

\section{To cite this version:}

Mehdi Bouatou, Rishav Harsh, Cyril Chacon, Yann Girard, Vincent Repain, et al.. Black Phosphorus for Directed Molecular Assembly with Weak Electronic Coupling. Advanced Materials Interfaces, 2021, pp.2101644. 10.1002/admi.202101644 . hal-03397109

\section{HAL Id: hal-03397109 \\ https://hal.science/hal-03397109}

Submitted on 22 Oct 2021

HAL is a multi-disciplinary open access archive for the deposit and dissemination of scientific research documents, whether they are published or not. The documents may come from teaching and research institutions in France or abroad, or from public or private research centers.
L'archive ouverte pluridisciplinaire HAL, est destinée au dépôt et à la diffusion de documents scientifiques de niveau recherche, publiés ou non, émanant des établissements d'enseignement et de recherche français ou étrangers, des laboratoires publics ou privés. 


\title{
Black Phosphorus for directed molecular assembly with weak elec- tronic coupling
}

\author{
Mehdi Bouatou Rishav Harsh Cyril Chacon Yann Girard Vincent Repain Amandine Bellec Sylvie \\ Rousset Alexander Smogunov Yannick J. Dappe Jérôme Lagoute*
}

M. Bouatou, R. Harsh, C. Chacon, Y. Girard, V. Repain, A. Bellec, A. Rousset, J. Lagoute

Laboratoire Matériaux et Phénomènes Quantiques, CNRS-Université de Paris, 10 rue Alice Domon et Léonie Duquet, 75205 Paris Cedex 13, France.

jerome.lagoute@u-paris.fr

R. Harsh

Donostia International Physics Center (DIPC), Paseo Manuel de Lardizábal 4, 20018 San Sebastián, Spain A. Smogunov, Y. J. Dappe

SPEC, CEA, CNRS, Université Paris-Saclay, CEA Saclay, 91191 Gif-sur-Yvette Cedex, France

Keywords: scanning tunneling microscopy, black phosphorus, molecules, density functional theory

The combination of two-dimensional materials with organic molecules offers the possibility to obtain low dimensional hybrid materials with tailored properties. Black phosphorus is a monoelemental semiconducting material with a non-planar puckered atomic structure. Here, we study porphyrin molecules on black phosphorus and show that they self-assemble with an epitaxial relationship indicating that the molecule-surface interaction largely dominates the molecule-molecule interaction. The atomic structure of black phosphorus is found to be at the origin of the substrate driven self-assembly. Despite this strong interaction with molecules, the electronic coupling is found to be weak allowing the molecules to maintain the properties of their gas phase, which is the usual behaviour for van der Waals materials. Therefore, the combination of the peculiar puckered structure with the van der Waals nature of black phosphorus provides this material with the ability to interact strongly enough with adsorbed molecules to drive their assembly but weakly enough to keep their electronic properties intact.

\section{Introduction}

The combination of organic molecules with two-dimensional (2D) materials provides a rich playground for the realization of hybrid materials with improved performances beyond the pristine materials. The molecule-substrate interface is the place where several physical phenomena occur such as charge transfer [1], gap renormalization [2], self-assembly [3], redox reactions [4]. Mastering these interactions would open the route toward interfacial engineering with various potential applications. Molecule-graphene hybrids have been used to create a bandgap in graphene with potential applications in graphene based electronic devices [5]. The functionalization of graphene with metalloporphyrins has been demonstrated to improve the performances of graphene based photodetectors and chemical sensors [6]. On black phosphorus (BP), non-covalent functionalization has been achieved with expected applications in electronics, optoelectronics, energy storage, sensors, and fillers for composite reinforcement [7]. Non-covalently or covalently bonded molecules on $\mathrm{BP}$ have been used for surface passivation $[8,9,10,11]$ together with the formation of ohmic contact and charge transfer that are ideal for BP based transport devices [12]. On 2D van der Waals materials, the molecule-surface interaction is generally weak [13], which has two major consequences. Firstly, the weak electronic coupling allows the molecules to maintain the electronic properties of their gas phase [13]. Secondly, molecular assembly on these materials can occur independently of the structure of the 2D material, except when the properties of the 2D materials are modified by the interaction with a substrate which often leads to the formation of moiré patterns $[14,15]$. Indeed, molecular assembly on surfaces depends on the interplay between molecule-molecule and moleculesurface interaction $[3,16,13]$. On non-interacting surfaces, the assembly is driven by intermolecular interactions which determine the molecular lattice parameters $[17,18,19]$. On interacting surfaces, the molecule-substrate interaction dominates and the substrate governs the assembly [20, 21, 22, 23]. In principle, on weak interacting surfaces, the forces driving the self-assembly and the electronic coupling are not directly linked since the former involves electrostatic and van der Waals interaction and the later involves hopping integral between molecules and surfaces wavefunctions. However, the case of interacting surfaces, where the substrate drives the molecular assembly is generally found on metallic surfaces, 
where the electronic coupling is non negligible. On van der Waals materials, the molecule-substrate interaction force is weak, and the electronic interaction is weak. An intermediate regime where a weak electronic coupling would combine with a strong adsorption force would allow to govern the molecular assembly by the substrate geometry while preserving their electronic properties. So far, interfacial effects on molecule/2D hybrids have been explored at the atomic scale with scanning tunneling microscopy (STM) on various substrates including graphene, hexagonal boron nitride and transition metal dichalcogenide [24, 13]. However, little is known for molecules on black phosphorus (BP). In contrast with other 2D materials, black phosphorus is a van der Waals material with a non-planar puckered structure. Its physical properties have been thoroughly investigated at the atomic scale by STM $[25,26]$ revealing anisotropic structural and electronic properties. Here, we study the interaction of iron tetraphenylporphyrin (FeTPP) molecules with BP. We show that the puckered nature of the atomic structure of BP provides to this material the property to drive the self-assembly process of organic molecules leading to a commensurable $3 \times 4$ molecular lattice. We evidence the weak electronic interaction between FeTPP and BP by comparing the properties of FeTPP/BP with FeTPP/graphene, that serves as a reference for non-interacting 2D material, and combining STM and density functional theory (DFT) calculations. Our results show that the van der Waals nature of $\mathrm{BP}$ combined with its non-planar structure gives to BP the property to have a strong interaction with molecules that drives the molecular assembly, while preserving the gas phase electronic properties of the molecules.

\section{Results and discussion}

A monolayer of FeTPP on BP forms a rectangular lattice as shown in Figure 1a. There are two types of molecules characterized by a bright or dark center. The molecules with a bright center correspond to FeTPP with a chlorine bonded to the Fe atom forming a FeTPP-Cl molecule [27, 28]. Those with a dark center correspond to FeTPP. We focus our study on FeTPP only, since the spectroscopic measurements on FeTPP $-\mathrm{Cl}$ often induce removal of the $\mathrm{Cl}$ ion and tip modification [29]. The unit cell vectors of the molecular lattice have lengths $1.25 \pm 0.01 \mathrm{~nm}$ along the [100] direction and $1.31 \pm 0.01 \mathrm{~nm}$ along the [010] direction (see more examples in supporting information Figure S1) which are smaller than the intermolecular distance obtained in FeTPP layers (and other TPP molecules) on other surfaces (see supporting information Figure S2). Indeed, on $\mathrm{Au}(111)$ FeTPP arrange in a rectangular lattice with a periodicity of $1.40 \pm 0.05 \mathrm{~nm}$ and $1.55 \pm 0.05 \mathrm{~nm}$ [27], on $\mathrm{Ag}(111)$ it forms a square lattice with a period of $1.43 \pm 0.01 \mathrm{~nm}$ [30], on graphene [28] we have found a square lattice with a periodicity of $1.4 \pm 0.02 \mathrm{~nm}$ (see supporting information Figure S3). Generally, tetraphenylporphyrin molecules adopt a square lattice with a period of $1.40 \mathrm{~nm}$ irrespective of the presence and nature of a central metal atom [31, 18]. Here the distance between adjacent molecules is shorter than on metallic substrates or graphene, which is due to the interaction with $\mathrm{BP}$ that forces the molecules to follow the BP atomic lattice. As a consequence, the density of TPP molecules on BP is increased by $20 \%$ with respect to TPP on other substrates. This could be advantageous in TPP based data storage systems such as $\mathrm{H}_{2} \mathrm{TPP}$ on surfaces. Indeed it has been shown recently that the control of tautomerisation in self-assembled $\mathrm{H}_{2}$ TPP molecules on graphene allows to store binary information on each molecule [32]. The increase of molecular density on $\mathrm{BP}$ can be used to increase the storage density on such systems. It can be noted that the molecular density found here is also higher than tin phtalocyanine $(\mathrm{SnPc})$ that have been used as molecular lattice for data storage on graphene even though single Pc molecules have a smaller size than TPP derivatives [33]. Moreover, due to the epitaxial relationship, TPP derivatives on BP form an addressable system where the position of the molecules is predetermined by the atomic lattice of the substrate. Another implication of the increased molecular density on BP is the potential gain of efficiency in hybrids materials involving TPP derivatives for catalysis. FeTPP is an efficient catalyst for $\mathrm{O}_{2}$ reduction [34] and $\mathrm{CO}_{2}$ reduction [35] reactions. The immobilization of porphyrin on $2 \mathrm{D}$ materials has been shown to improve the catalytic activity of porphyrin compounds for $\mathrm{CO}_{2}$ reduction [36]. The increase of in-plane density may further improve the performances of such hybrid materials. This comes from the interaction with the BP substrate which forces the molecules to follow the BP atomic lattice. 
In order to understand the role of the BP lattice on the molecular assembly, one has to determine the relationship between the molecules and the underlying $\mathrm{BP}$ atomic lattices. Usually, this information is not accessible by STM since the substrate underneath the molecules cannot be visualized. Here, to be able to visualize the substrate below the molecular layer, we have removed all the molecules using molecular manipulation on large scale with the STM tip. We have scanned the surface several times with a reduced tip-sample distance in order to sweep away the molecules. After several scanning with various setpoint parameters, we were able to recover a good tip state allowing to visualize the atomic lattice of the BP on the same area. This procedure is similar to the one that we have used in our previous works on graphene $[37,28,4]$. The image of the BP surface shows the phosphorus zigzag rows (Figure 1b). Indeed, a single BP layer has a puckered structure made of two planes of phosphorus atoms. In the following we will refer to top and bottom $\mathrm{P}$ atoms in a BP layer depending on whether the atoms are in the top (facing the vacuum side) or bottom (facing the bulk side) plane. The atomically resolved STM image corresponds to the top $\mathrm{P}$ atoms that form zigzag lines. The measured BP unit cell vectors length are $|\vec{a}|=4.17 \pm 0.1 \AA$ along the armchair direction $[100]$ and $|\vec{b}|=3.27 \pm 0.1 \AA$ along the zigzag direction [010]. Consequently, the FeTPP layer remarkably matches the BP atomic lattice with a ratio of 3 along the armchair direction and 4 along the zigzag direction, leading to a $3 \times 4$ pattern. This epitaxial relation is further evidenced in the FFT image of Figure 1c that reveals the periodicity of BP and FeTPP. The FFT was obtained from a combined image made of the addition of the images of Figures 1a and 1b. This procedure allows to visualize in one single image the FFT of the BP and the FeTPP lattices and to highlight the $3 \times 4$ pattern. This epitaxial relationship indicates that the molecule-surface interaction is the dominating force that stabilizes the molecular lattice. The relaxed structure of FeTPP on BP from our ab initio calculations reveals that the molecules adsorb on the surface with the Fe atom located above the topmost zigzag row of BP as shown in Figure 1d. The molecular axis represented in Figure $1 \mathrm{~d}$ (connecting two opposite phenyl groups) is rotated by an angle of $35.5^{\circ}$ with respect to the molecular lattice vector which is aligned with the zigzag direction. This azimuthal angle corresponds to an intermolecular interaction of "T-type" consisting of an edge to face arrangement of the phenyl groups [31]. In order to understand the driving force for the molecular self-assembly on BP we have performed ab initio calculations including van der Waals forces. First, we have determined the adsorption energy of one FeTPP molecule on the BP surface. To this end, we have considered a $3 \times 4$ unit cell of BP composed of 3 atomic layers. The FeTPP molecule has been placed on top of the BP slab, and the full system has been optimized until the forces went below $0.1 \mathrm{eV} / \AA$ using the localized-orbitals basis set DFT code Fireball [38]. From the optimized configuration, we have then calculated the interaction energy between the FeTPP molecule and the BP (defined by the difference in total energies $E_{F e T P P+B P}-E_{F e T P P}-$ $\left.E_{B P}\right)$ at different heights from the BP surface. Van der Waals interactions have been taken into account through the use of a specific formalism, the LCAO-S ${ }^{2}+\mathrm{vdW}$ approach [39], previously used for similar systems $[40,41]$. The calculated interaction energies are presented in Figure 2a. We compare this interaction energy with the case of FeTPP on graphene that we take as a reference for weakly coupled FeTPP [28]. The optimized equilibrum configuration on BP is presented in Figure 2b. As it can be clearly seen, the adsorption energy on BP is much higher (about $2.4 \mathrm{eV}$ ) than the one obtained on graphene (about $1.5 \mathrm{eV}$ ). Note also that the most stable position of the molecule on BP is obtained for the molecule center on a zigzag row of top P atoms. Secondly, we have determined the interaction energy of a molecule in the network of its neighboring molecules. Using the same approach as for the interaction with the BP surface, we have considered a set of nine molecules isolated in a plane, and we have calculated the interaction of the central molecule with its neighbors as a function of the intermolecular distance. The result is represented in Figure 2c, and the corresponding molecular network at equilibrium is represented in Figure $2 \mathrm{~d}$. The obtained interaction energy is about $0.78 \mathrm{eV}$ per molecule, namely three times less than the interaction of the molecule with the BP surface. Also the intermolecular distance in a free standing layer obtained in this calculations is $13.85 \AA$ in one direction and $14.35 \AA$ in the other one, in both cases much bigger than the lattice parameters determined experimentally for the molecules on BP. Therefore, the molecular network is constrained upon adsorption on the BP surface. This constraint can be roughly estimated energetically at around $2.5 \mathrm{eV}$ loss in the interaction energy between molecules, when extrapo- 
lating the curve in Figure 2c. This could appear to be huge, but it has to be compared to the interaction energy with the substrate presented in Figure 2a which is about $2 \mathrm{eV}$. Moreover, we have to stress that the results presented in Figure 2c arise from a static calculation, starting from fully optimized isolated molecules, arranged in a network and displaced rigidly without further optimization. Obviously, the very high repulsion found in Figure 2c would be attenuated through a further optimization, leading to a more important substrate-molecule interaction than the molecule-molecule repulsion. In other words, these findings fully confirm the fact that the BP substrate stabilizes the molecular network in a constrained phase. The surface-driven molecular organization is due to the unique puckered atomic structure of BP. Indeed, along the armchair direction, the van der Waals interaction results in high energy gain when the molecule locates above the top $\mathrm{P}$ atoms because the molecule-phosphorus distance is much shorter as compared to the bottom $\mathrm{P}$ atoms. This allows to understand that along the armchair direction, the molecules match the BP atomic lattice. Along the zigzag direction, a first intuitive argument would be that all the $\mathrm{P}$ atoms are at the same height and therefore the molecules should not be constrained in that direction. In order to gain a deeper insight into the driving force of the self-organization, we plot the charge redistribution between BP and FeTPP in Figure 2e,f. In Fig 2e the isosurface of the charge density difference shows that above $\mathrm{BP}$ the charge redistribution is dominated by the top $\mathrm{P}$ atoms. This charge redistribution is a consequence of the puckered structure of $\mathrm{BP}$ that leads to a reduced interaction between bottom $\mathrm{P}$ atoms and FeTPP as compared to the top $\mathrm{P}$ atoms. This is in line with the argument given above that the van der Waals interaction is weaker for the bottom $\mathrm{P}$ atoms. Strikingly, in contrary to the first intuitive expectation, a strong corrugation in the charge redistribution is also present along the zigzag direction (Figure 2f). This indicates that the molecule 'see' a non uniform potential also along the zigzag line. This is a consequence of the atomic structure of BP, not only the buckling that occurs in the armchair direction, but also the large interatomic distance between the top $\mathrm{P}$ atoms. Indeed, in the [010] direction, the P atoms are separated by a distance of $3.3 \AA$ which is larger than the standard P-P bond-length. As a consequence a strong variation of the potential occurs in that direction and the van der Waals interaction is non uniform leading to a constrain in the molecular assembly in the zigzag direction. Therefore, the constrained self-assembly that occurs on BP is a consequence of the geometry of the atomic lattice that leads to strong periodic variations of the van der Waals interaction between the molecule and the surface along the armchair and zigzag direction. On other 2D materials such as graphene, the shorter interatomic distance prevents such strong variations of the force interaction and charge redistribution leading to a smooth potential variations on the surface and to molecular assembly driven by intermolecular forces which is opposite to the molecule-surface dominating interaction on BP. The BP appears to be an interacting substrate that drives the organization of adsorbed molecules. Therefore a strong electronic interaction between BP and FeTPP is expected. Surprisingly this is not the case. The first information on the electronic properties of FeTPP on BP is obtained by visualizing the HOMO and LUMO states from STM topographical images (Figure 3). The central part of the molecules appears as a protrusion at negative bias $(-1 \mathrm{~V}$, Figure $3 \mathrm{a}, \mathrm{c})$ and as a depletion at positive bias $(+2 \mathrm{~V}$, Figure $3 \mathrm{~b}, \mathrm{~d}$ ). Note that at negative bias both the FeTPP-Cl molecules and FeTPP appear with a protrusion at the center and that FeTPP appear brighter than FeTPP-Cl unlike what is observed at positive bias. The images of FeTPP on BP are very similar to the ones of FeTPP on graphene [28] at $-1 \mathrm{~V}$ and $+2 \mathrm{~V}$. On graphene the image at $-1 \mathrm{~V}$ arises from the HOMO state localized on the Fe atom, and the image at $+2 \mathrm{~V}$ arises from the LUMO and higher unoccupied states localized on the organic part. The same trend is observed here, on BP. A close look at the images in Figure 3c,d reveals four lobes at the periphery of the molecule corresponding to the phenyl groups (as indicated by the molecular model superimposed to the experimental images). The corresponding azimuthal angle $\theta$ with the [010] axis of BP measured in Figure $3 \mathrm{c}$ is $31 \pm 4^{\circ}$, close to the value obtained in our calculation. From these topographical images we conclude that the HOMO state is localized on the iron atom, and that the azimuthal angle is in agreement with the calculations, which corresponds to a T-type interaction between molecules. More insight is given by the spectroscopy measured at different positions above a FeTPP molecule. In Figure 4, we show the spectra measured above the Fe atom, a pyrrole group, and a phenyl group. Two peaks are visible at negative bias corresponding to the $\mathrm{HOMO}-1$ at $-1.38 \mathrm{~V}$, and the HOMO at $-1 \mathrm{~V}$. 
At positive bias, a broad peak appears for which 2 contributions can be distinguished at $1.95 \mathrm{~V}$ and $2.2 \mathrm{~V}$. The measured HOMO-LUMO gap is $2.95 \mathrm{~V}$. The peak of the HOMO state dominates the spectrum at the center of the molecule, revealing that the HOMO state is localized on the Fe atom. The HOMO-1 and the peaks at positive bias are localized on the pyrrole and phenyl groups. The localization of the states can be further evidenced from $d I / d V$ maps which are shown in Figure 4d. These electronic properties are very similar to those of FeTPP on graphene [28] (see supporting information Figure S4) where a HOMO-LUMO gap of $2.9 \mathrm{~V}$ was measured and the localization of the molecule electronic states was similar. A noticeable difference is found at positive bias where 3 sharp peaks could be clearly distinguished on graphene around the LUMO state, whereas a broad peak is observed here and only two contributions can be distinguished. This discrepancy comes from a difference in the electronic properties of graphene and BP. While the local density of states (LDOS) of graphene increases linearly with the energy and symmetrically around the Dirac point, the LDOS of BP exhibits a bandgap but also a strong asymmetry with a strong increase of the measured LDOS at positive sample bias voltage as shown in Figure 4c. Around the Fermi level, the spectrum shows a gap of $0.3 \mathrm{~V}$ with a valence band edge close to the Fermi level indicating that the BP is $p$-doped. The measured gap corresponds to the expected value for bulk BP and the spectroscopy is in agreement with previous STM reports [25, 26]. At larger bias, we observe the presence of a peak at $1.5 \mathrm{~V}$. The asymmetry of the $d I / d V$ spectrum with a large value at positive bias explains that the molecular states around the LUMO undergo a large broadening as compared to the states at negative bias. This explains why on BP the molecular spectrum around the LUMO state is not as resolved as on graphene.

To interpret the experimental spectra we have carried out DFT calculations using plane wave electronic structure package Quantum ESPRESSO (QE) within local density approximation (in Perdew-Zunger parametrization) for exchange-correlation potential. We have applied a Hubbard-like correction of $U$ $=5 \mathrm{eV}$ to Fe d orbitals, as in our previous paper for FeTPP on graphene and $\mathrm{Au}(111)$ [28], in order to compare to those results. The calculations for FeTPP on three-layers BP slab reveal that the lowest energy state has a spin state $\mathrm{S}=1$ with a $\left(d_{x y}\right)^{2}\left(d_{x z}\right)^{1}\left(d_{y z}\right)^{1}\left(d_{z^{2}}\right)^{2}$ configuration as demonstrated in Figure 5 where total, molecular and Fe d-orbital resolved DOS are presented. This state is generally found to be the ground state of isolated FeTPP $[42,43,44,45]$. On BP, this state is lower in energy by about 80 meV compared to other $\mathrm{S}=1$ states with configurations $\left(d_{x y}\right)^{2}\left(d_{x z}\right)^{2}\left(d_{y z}\right)^{1}\left(d_{z^{2}}\right)^{1}$ or $\left(d_{x y}\right)^{2}\left(d_{x z}\right)^{1}\left(d_{y z}\right)^{2}\left(d_{z^{2}}\right)^{1}$, which is similar to the case of graphene [28] (but different from $\mathrm{Au}(111)$ ) indicating relatively weak electronic hybridization of molecular states with BP substrate. One can observe, in particular, that HOMO, located at about $1 \mathrm{eV}$ below the Fermi energy, is heavily dominated by the mentioned above Fe (spin down) $d_{z^{2}}$ orbital (shown in green) which extends largely in the vacuum and can be therefore readily probed by STM, in agreement with experimental observations. Unocuppied molecular states are, on the contrary, localized on molecular skeleton (which also agrees with the experimental STS spectra) and are non spin-polarized. The present results are very close to those for FeTPP on graphene and to the fundamental state of a free FeTPP molecule [28]. These electronic properties, together with the self-assembly described above show that $\mathrm{BP}$ is a material that interacts strongly enough with molecules to govern their assembly, but weakly enough to preserve their intrinsic electronic properties. In that respect, BP is a unique material that can combine these 2 properties which usually exclude each other.

\section{Conclusion}

In conclusion, we have studied the interaction of black phosphorus with FeTPP molecules. The molecules self-assemble on the surface following the periodicity of BP. This assembly is due to the molecule-surface interaction that dominates the molecule-molecule interaction. The unique structure of BP combining zigzag and armchair rows leads to an energy gain of the system due to van der Waals interaction that dominate the energy loss induced by the reduced intermolecular distances. As a consequence, the molecules adopt a dense lattice to fit with the periodicity of BP. The elecronic spectra of the adsorbed molecules reveal a $\mathrm{S}=1$ groundstate similar to the one previously observed on graphene and to the free molecule. These results show that BP allows to drive molecular self-assembly while preserving the electronic prop- 
erties of the molecules. This opens new avenues for functional surfaces based on 2D materials.

\section{Experimental Section}

Black phosphorus samples were purchased from HQ Graphene. The samples were cleaved under ultrahigh vacuum conditions. All STM measurements were performed with a low-temperature STM apparatus (Omicron) working at $4.6 \mathrm{~K}$ at a pressure lower than $1 \times 10^{-10}$ mbar. The $\mathrm{d} I / \mathrm{d} V$ spectra were acquired using a lock-in detector at a frequency of ca. $823 \mathrm{~Hz}$ and a modulation amplitude of $35 \mathrm{mV}$. The measurements were performed with an electrochemically etched tungsten tip. Before measuring on graphene, the tip was calibrated on a $\mathrm{Au}(111)$ surface until it showed the Shockley surface state feature in the spectroscopic measurements.

\section{Supporting Information}

Supporting Information is available from the Wiley Online Library or from the author.

\section{Acknowledgements}

This project received funding from the European Union's Horizon 2020 research and innovation programme under Grant Agreement No. 766726. DFT calculations have been performed using HPC resources from GENCI-CINES (Grant A0100910407).

\section{References}

[1] X. Lu, M. Grobis, K. H. Khoo, S. G. Louie, M. F. Crommie, Phys. Rev. B 2004, 70, 11115418.

[2] J. D. Sau, J. B. Neaton, H. J. Choi, S. G. Louie, M. L. Cohen, Phys. Rev. Lett. 2008, 101, 2 026804.

[3] R. Otero, J. M. Gallego, A. L. V. de Parga, N. Martín, R. Miranda, Adv. Mater. 2011, 235148.

[4] M. Bouatou, S. Mondal, C. Chacon, F. Joucken, Y. Girard, V. Repain, A. Bellec, S. Rousset, S. Narasimhan, R. Sporken, Y. J. Dappe, J. Lagoute, Nano Lett. 2020, 206908.

[5] X. Xu, C. Liu, Z. Sun, T. Cao, Z. Zhang, E. Wang, Z. Liu, K. Liu, Chem. Soc. Rev. 2018, 47, 9 3059.

[6] S. Pyo, J. Choi, J. Kim, Nanotechnology 2019, 30, 21215501.

[7] G. Abellán, V. Lloret, U. Mundloch, M. Marcia, C. Neiss, A. Görling, M. Varela, F. Hauke, A. Hirsch, Angew. Chem. 2016, 128, 4714777.

[8] V. V. Korolkov, I. G. Timokhin, R. Haubrichs, E. F. Smith, L. Yang, S. Yang, N. R. Champness, M. Schröder, P. H. Beton, Nat. Commun. 2017, 8, 11385.

[9] V. Artel, Q. Guo, H. Cohen, R. Gasper, A. Ramasubramaniam, F. Xia, D. Naveh, npj $2 D$ Mater. Appl. 2017, 16 .

[10] Y. Abate, D. Akinwande, S. Gamage, H. Wang, M. Snure, N. Poudel, S. B. Cronin, Adv. Mater. 2018, 301704749.

[11] R. Guo, Y. Zheng, Z. Ma, X. Lian, H. Sun, C. Han, H. Ding, Q. Xu, X. Yu, J. Zhu, W. Chen, Appl. Surf. Sci. 2019, 496143688.

[12] S. Wang, J. Li, Y. Zhao, B. Liu, P. Yuan, J. Wei, J. Zhang, H. Xie, D. Niu, M. Long, Appl. Phys. Lett. 2020, 117, 6061602.

[13] A. Kumar, K. Banerjee, P. Liljeroth, Nanotechnology 2017, 28082001. 
[14] H. G. Zhang, J. T. Sun, T. Low, L. Z. Zhang, Y. Pan, Q. Liu, J. H. Mao, H. T. Zhou, H. M. Guo, S. X. Du, F. Guinea, H.-J. Gao, Phys.Rev. B 2011, 84, 24245436.

[15] D. Maccariello, M. Garnica, M. A. Nino, C. Navio, P. Perna, S. Barja, A. L. Va'zquez de Parga, R. Miranda, Chem. Mater. 2014, 26, 92883.

[16] S. Whitelam, Adv. Mater. 2015, 275720.

[17] P. Järvinen, S. K. Hämäläinen, K. Banerjee, P. Häkkinen, M. Ijäs, A. Harju, P. Liljeroth, Nano Lett. 2013, 133199.

[18] V. D. Pham, J. Lagoute, O. Mouhoub, F. Joucken, V. Repain, C. Chacon, A. Bellec, Y. Girard, S. Rousset, ACS Nano 2014, 89403.

[19] H.-Z. Tsai, A. A. Omrani, S. Coh, H. Oh, S. Wickenburg, Y.-W. Son, D. Wong, A. Riss, H. S. Jung, G. D. Nguyen, G. F. Rodgers, A. S. Aikawa, T. Taniguchi, K. Watanabe, A. Zettl, S. G. Louie, J. Lu, M. L. Cohen, M. F. Crommie, ACS nano 2015, 912168.

[20] S. Lukas, G. Witte, C. Wöll, Phys. Rev. Lett. 2002, 88.

[21] M. G. Betti, P. Gargiani, C. Mariani, R. Biagi, J. Fujii, G. Rossi, A. Resta, S. Fabris, S. Fortuna, X. Torrelles, M. Kumar, M. Pedio, Langmuir 2012, 2813232.

[22] L. Massimi, M. Angelucci, P. Gargiani, M. G. Betti, S. Montoro, C. Mariani, J. Chem. Phys. 2014, 140244704.

[23] T. Bathon, P. Sessi, K. A. Kokh, O. E. Tereshchenko, M. Bode, Nano Lett. 2015, 152442.

[24] J. M. MacLeod, F. Rosei, Small 2014, 101038.

[25] B. Kiraly, N. Hauptmann, A. N. Rudenko, M. I. Katsnelson, A. A. Khajetoorians, Nano Lett. 2017, 173607.

[26] Z. Qiu, H. Fang, A. Carvalho, A. Rodin, Y. Liu, S. J. Tan, M. Telychko, P. Lv, J. Su, Y. Wang, et al., Nano lett. 2017, 17, 116935.

[27] T. G. Gopakumar, H. Tang, J. Morillo, R. Berndt, J. Am. Chem. Soc. 2012, 13411844.

[28] M. Bouatou, R. Harsh, F. Joucken, C. Chacon, V. Repain, A. Bellec, Y. Girard, S. Rousset, R. Sporken, F. Gao, M. Brandbyge, Y. J. Dappe, C. Barreteau, A. Smogunov, J. Lagoute, J. Phys. Chem. Lett. 2020, 119329.

[29] K. Scheil, M. Gruber, M. Ondrácek, R. Berndt, J. Phys. Chem. C 2020, 12426889.

[30] W. Auwärter, A. Weber-Bargioni, S. Brink, A. Riemann, A. Schiffrin, M. Ruben, J. V. Barth, ChemPhysChem 2007, 8, 2250.

[31] F. Buchner, I. Kellner, W. Hieringer, A. Görling, H.-P. Steinrück, H. Marbach, Phys. Chem. Chem. Phys. 2010, 1213082.

[32] R. Harsh, F. Joucken, C. Chacon, V. Repain, Y. Girard, A. Bellec, S. Rousset, R. Sporken, A. Smogunov, Y. J. Dappe, J. Lagoute, J. Phys. Chem. Lett. 2019, 10, 216897.

[33] M. Bouatou, C. Chacon, F. Joucken, Y. Girard, V. Repain, A. Bellec, S. Rousset, R. Sporken, C. González, Y. J. Dappe, J. Lagoute, J. Phys. Chem. C 2020, 124, 2815639.

[34] M. L. Pegis, D. J. Martin, C. F. Wise, A. C. Brezny, S. I. Johnson, L. E. Johnson, N. Kumar, S. Raugei, J. M. Mayer, J. Am. Chem. Soc. 2019, 14, 208315.

[35] H. Rao, L. C. Schmidt, J. Bonin, M. Robert, Nature 2017, 548, 766574. 
[36] M. Zhu, C. Cao, J. Chen, Y. Sun, R. Ye, J. Xu, Y.-F. Han, ACS Appl. Energy Mater. 2019, 2, 4 2435.

[37] V. D. Pham, F. Joucken, V. Repain, C. Chacon, A. Bellec, Y. Girard, S. Rousset, R. Sporken, M. C. dos Santos, J. Lagoute, Sci. Rep. 2016, 624796.

[38] J. P. Lewis, P. Jelínek, J. Ortega, A. A. Demkov, D. G. Trabada, B. Haycock, H. Wang, G. Adams, J. K. Tomfohr, E. Abad, H. Wang, D. A. Drabold, Phys. Status Solidi B 2011, 248, 91989.

[39] Y. J. Dappe, M. A. Basanta, F. Flores, J. Ortega, Phys. Rev. B 2006, 74205434.

[40] P. Sleczkowski, Y. J. Dappe, B. Croset, Y. Shimizu, D. Tanaka, R. Minobe, K. Uchida, E. Lacaze, J. Phys. Chem. C 2016, 120, 3922388.

[41] T. Knaak, C. González, Y. J. Dappe, G. D. Harzmann, T. Brandl, M. Mayor, R. Berndt, M. Gruber, J. Phys. Chem. C 2019, 123, 74178.

[42] J. P. Collman, J. Hoard, N. Kim, G. Lang, C. A. Reed, J. Am. Chem. Soc. 1975, 97, 102676.

[43] P. D. Boyd, D. A. Buckingham, R. F. McMeeking, S. Mitra, Inorg. Chem. 1979, 18, 123585.

[44] M.-S. Liao, S. Scheiner, J. Chem. Phys. 2002, 117, 1205.

[45] M.-S. Liao, J. D. Watts, M.-J. Huang, J. Phys. Chem. A 2007, 111, 265927. 

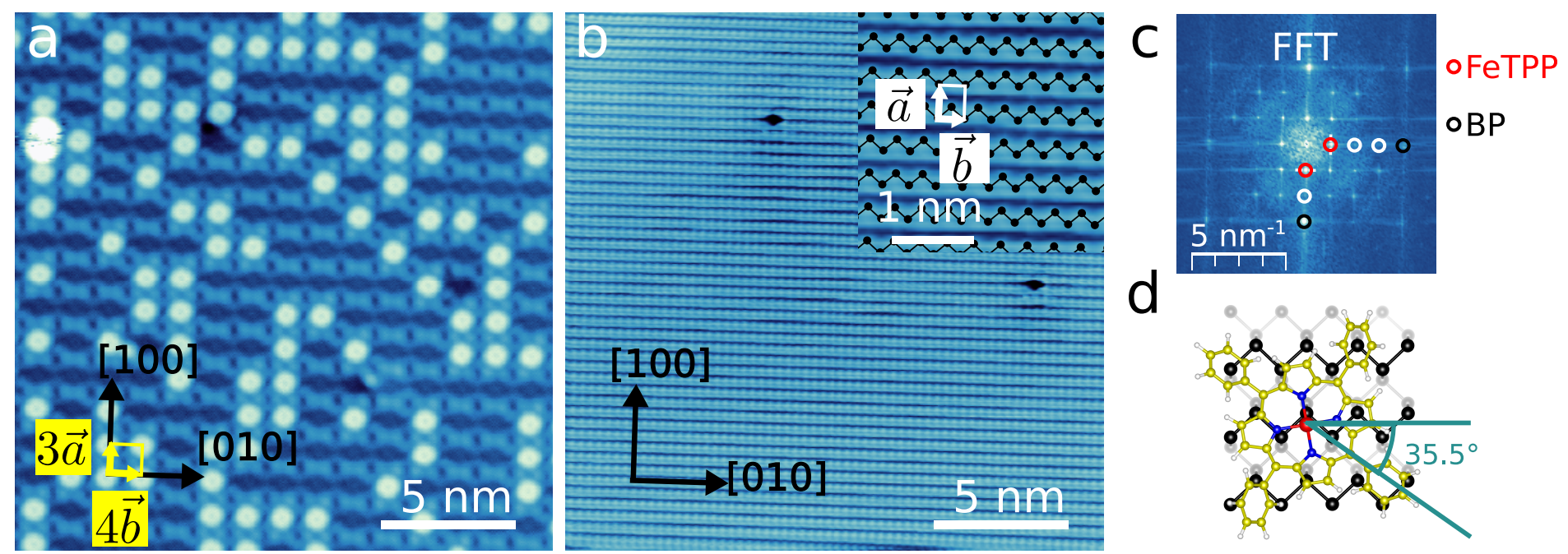

Figure 1: Assembly of FeTPP on BP. (a) STM image (2 V, 10 pA) of self-assembled FeTPP molecules adsorbed on BP. The black arrows indicate the crystallographic directions of BP, the yellow arrows and square indicate the unit cell of the FeTPP lattice. (b) STM image (-0.4 V, $200 \mathrm{pA})$ of the same area as in (a) after removing the molecules with the STM tip. The inset is a zoom of atomically resolved image with the BP unit cell indicated. (c) FFT of the superposition of the STM image of the FeTPP molecules with the image of the BP atomic structure. The peaks corresponding to the FeTPP lattice are marked with red circle, those of the BP atomic lattice are marked with black circles. Intermediate spots along the two main axis are indicated with white markers. (d) Relaxed structure of FeTPP on a BP monolayer. Color code: Black for phosphorus, yellow for carbon, blue for nitrogen, red for Fe, white for hydrogen.

a

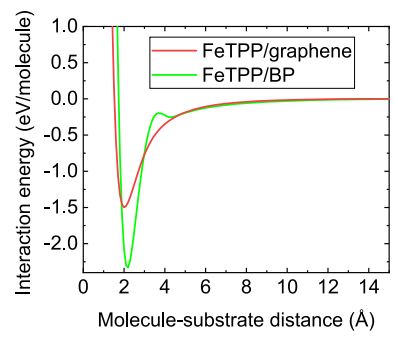

C

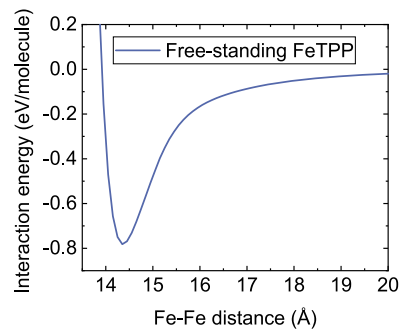

b

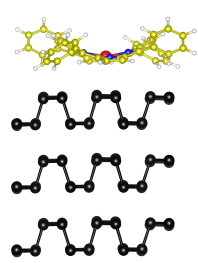

d

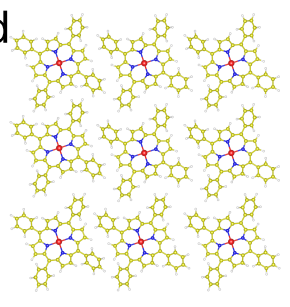

e

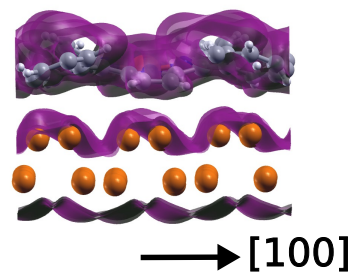

f

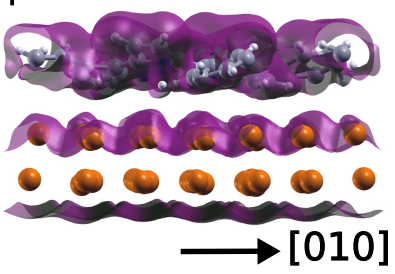

Figure 2: (a) Interaction energy of FeTPP on black phosphorus as a function of the molecule-surface distance, compared to the interaction energy for FeTPP on graphene. (b) Side view of the optimized structure of FeTPP on BP. (c) Interaction energy of one FeTPP molecule in the middle of the self-assembled network of its neighboring molecules, as a function of the Fe-Fe distance between neighboring molecules showing a minimum for a Fe-Fe distance of $14.35 \AA$. (d) Structure of the FeTPP layer considered for the interaction energy calculated in (c). (e,f) Side view of the charge density difference (isovalue $0.01 \mathrm{e})$. 

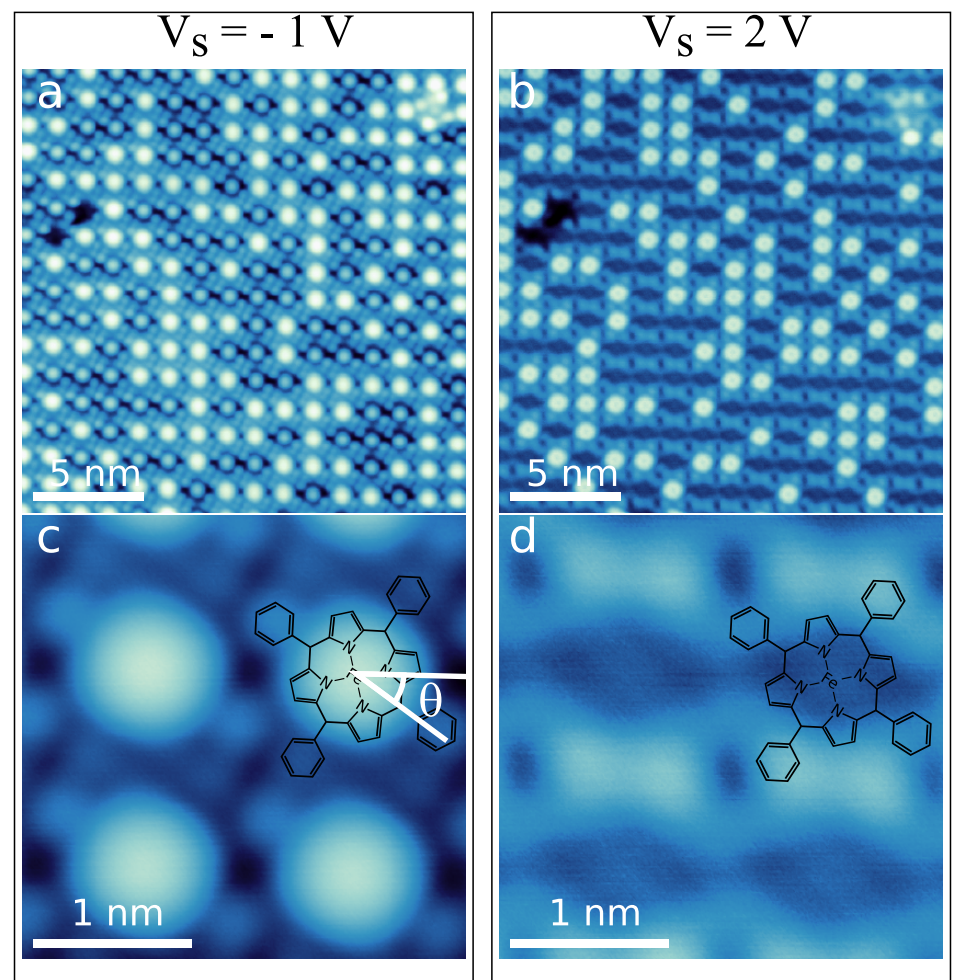

Figure 3: Bias voltage dependent STM images of FeTPP. STM images of FeTPP on BP measured at (a) -1 V, 10 pA and (b) $2 \mathrm{~V}, 10 \mathrm{pA}$. (c,d) Zoomed images of $(\mathrm{a}, \mathrm{b})$ on FeTPP without $\mathrm{Cl}$ atom with a superimposed model of FeTPP as a guide to the eye.
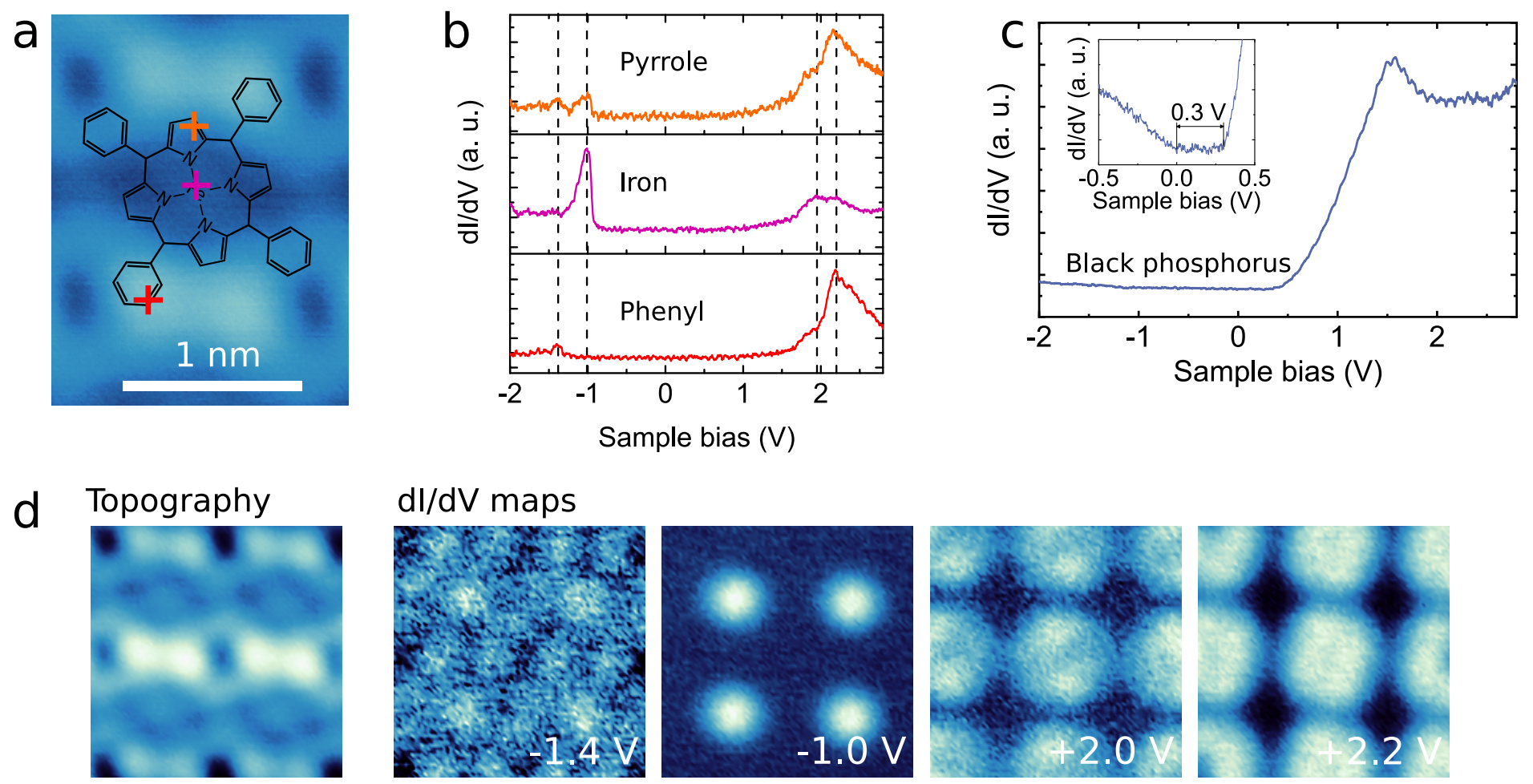

Figure 4: Electronic properties of FeTPP on BP. (a) STM image (2 V, $40 \mathrm{pA})$ of a FeTPP molecule with a model that serves as a guide to the eye. (b) $d I / d V$ spectra measured at the locations indicated by crosses in (a). (c) $d I / d V$ spectrum of BP. The inset is a close view in a small voltage range around the Fermi level showing the bandgap of BP. (d) Topography $(2 \mathrm{~V}, 60 \mathrm{pA})$ and $d I / d V$ maps of four FeTPP molecules on BP (image size $2.8 \mathrm{~nm} \times 2.8 \mathrm{~nm}$ ). 

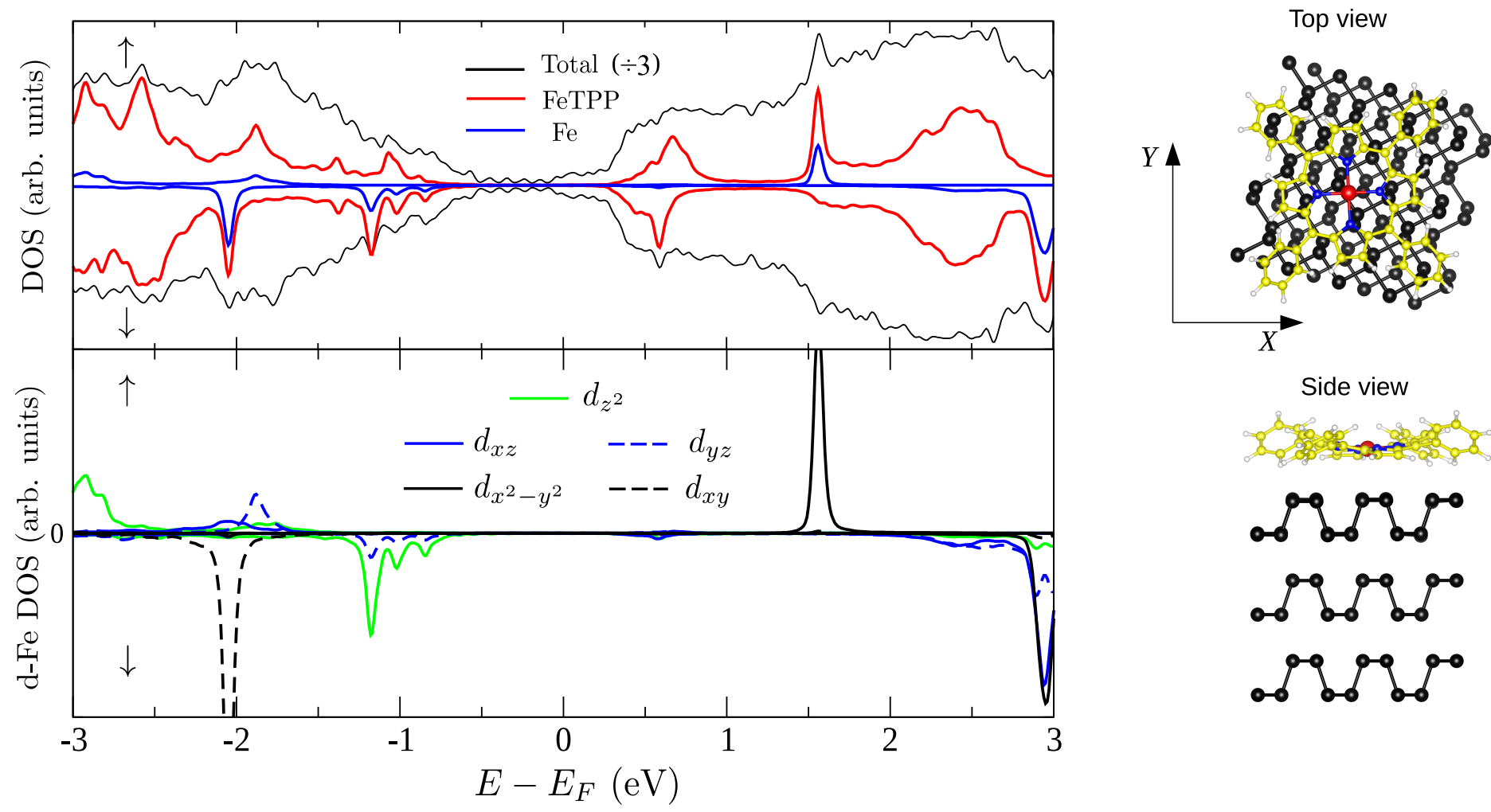

Side view
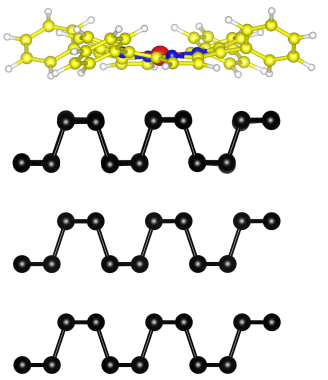

Figure 5: Spin-resolved DOS for FeTPP on three-layer slab of BP. Top: total DOS (black), as well as DOS projected on FeTPP (red) or on Fe (blue). Bottom: PDOS (projected DOS) on different Fe d-orbitals. The right panels show the relaxed geometry of the FeTPP on BP.

Table of Contents

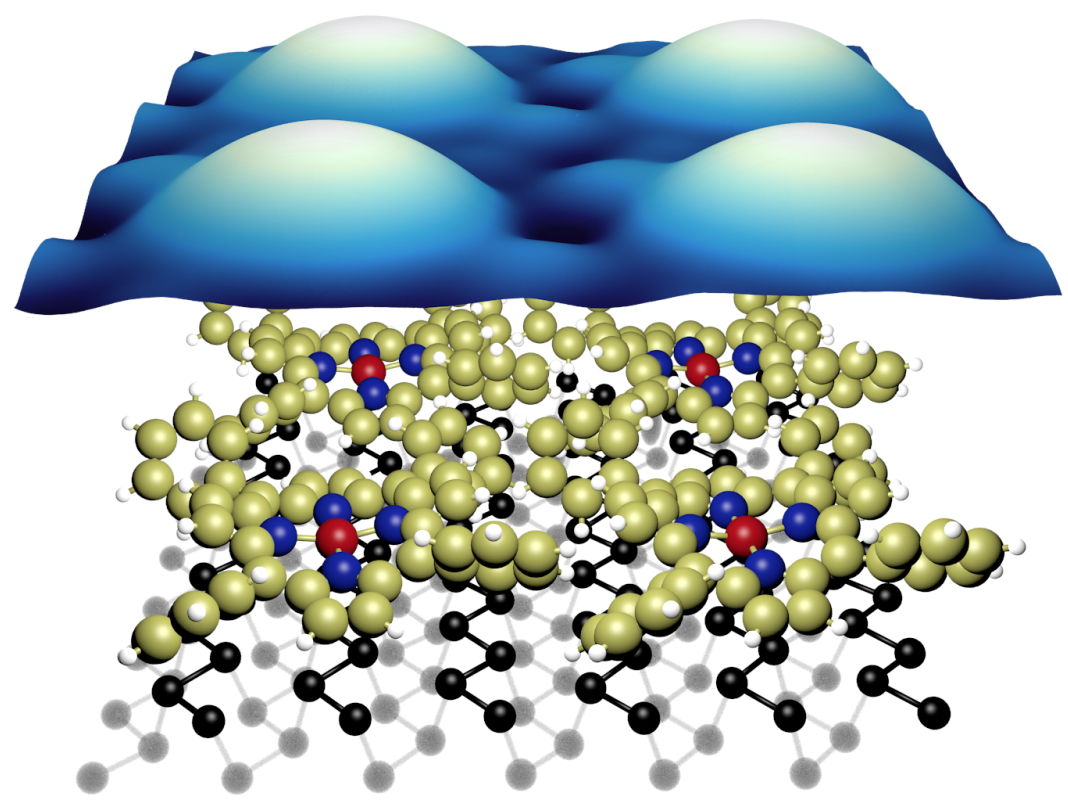

Iron tetraphenylporphyrin molecules on black phosphorus are studied by scanning tunneling microscopy. The epitaxial relation between molecules and black phorphorus, and the local spectroscopy reveals that the interaction of black phosphorus with molecules is strong enough to drive their assembly while the electronic coupling is weak enough to allow the molecules to maintain the electronic properties of their gas phase. 